\title{
Signal-to-Noise Ratio Improvement in BOTDA Using Balanced Detection
}

\author{
Alejandro Domínguez-López, Alexia López-Gil, Sonia Martín-López, and Miguel González-Herráez
}

\begin{abstract}
Brillouin optical time domain analysis (BOTDA) relies typically on the interaction among two counter-propagating waves: 1) a pulsed pump wave and 2) a modulated probe wave. The modulated probe wave has typically two sidebands, located at $\pm v_{B}$ with respect to the pump frequency. Conventional systems detect the time-resolved gain/loss by detecting only the upper/lower wavelength sideband. In this letter, we show that BOTDA can strongly benefit from the use of balanced detection among the two sidebands. In particular, the detected signal can be doubled while the noise only grows by a factor of $(2)^{1 / 2}$, leading to a $(2)^{1 / 2}$ signal-to noise ratio (SNR) increase. Moreover, any common-mode noise in the probe signal path (e.g., master laser noise, modulator drifts, and so forth) is eliminated, rendering the system more robust. We validate the principle by experimental results that highlight the benefits of the technique in terms of the SNR.
\end{abstract}

Index Terms-Brillouin scattering, distributed optic fiber sensor, balanced detection, temperature sensor.

\section{INTRODUCTION}

$\mathbf{I}$ N THE past few decades Brillouin-based distributed temperature and strain sensors have attracted the attention of both the academic and industrial sectors. In particular, BOTDA systems have been lately a trendy study area due to their numerous and well proofed applications and advantages, such as in monitoring large civil structures, energy transportation or environmental applications.

One of the main areas of research in BOTDA sensors focuses on the extension of the measuring range. To do so, it becomes necessary to increase the signal to noise ratio (SNR) of the sensor, in particular considering the reduced signal contrast at the far end of the fiber. Several techniques have been applied in order to achieve this goal, such as Raman amplification [1], pulse coding [2] or a combination of both [3]. Chopping techniques have also been proposed to increase the SNR in Brillouin optical correlation domain analysis [4].

Manuscript received October 7, 2013; revised November 20, 2013; accepted November 27, 2013. Date of publication December 5, 2013; date of current version January 21, 2014. This work was supported in part by the European Research Council through U-FINE under Grant 307441, in part by the Spanish Ministry of Science and Innovation under Projects TEC2009-14423-C02-01 and TEC2009-14423-C02-02, in part by the INTERREG SUDOE program ECOAL-MGT and in part by the Comunidad de Madrid under Project FACTOTEM-2. The work of S. Martín-López was supported by the Spanish Ministry of Science and Innovation through a Ramón y Cajal Contract.

The authors are with the Departamento de Electrónica, Universidad de Alcalá, Edificio Politécnico, Madrid 28871, Spain (e-mail: alejandro. dominguezl@uah.es; alexia.lopez@uah.es; sonia.martin@depeca.uah.es; miguelg@depeca.uah.es).

Color versions of one or more of the figures in this letter are available online at http://ieeexplore.ieee.org.

Digital Object Identifier 10.1109/LPT.2013.2293603
In this letter, we propose the use of a well-known detection technique to increase the SNR: the balanced detection. This detection procedure has resulted in remarkable improvements for several methods [5] in other areas of photonics, such as heterodyne detection [6], optical communications [7], [8] or biomedical applications [9]. We show that a balanced detection among the Stokes and anti-Stokes sidebands in a BOTDA can allow improving the results obtained over a conventional single-detector option.

\section{PRINCIPLES}

Stimulated Brillouin Scattering (SBS) features two side bands: the Stokes (gain) and Anti-Stokes (loss) bands [10], [11]. To take advantage of the SBS phenomenon, a single sideband (SSB) modulator can be used at the probe signal to work at Brillouin Gain frequency (Stokes band) or at Brillouin Loss frequency (Anti-Stokes band). However, making use of a dual sideband (DSB) modulator to generate both Stokes and Anti-Stokes bands, the effect of pump depletion can be effectively mitigated [12]. For this reason, DSB modulation turns out to be the preferred option in long-range setups. In conventional BOTDA, one of the two sidebands (Stokes or anti-Stokes) is filtered in detection and fed into a conventional photo-receiver for analysis.

In balanced detection, the receiver features two well matched photodiodes. Two light beams are fed into the corresponding photodiode and the obtained currents are subtracted. Ultimately, the remaining current difference is amplified through a trans-impedance amplifier. This technique allows for the detection of small signal variations over substantial DC levels. We show in this letter that this technique can be used advantageously in BOTDA by performing the balanced detection scheme using the Stokes and Anti-Stokes bands in each input diode. In this way, the differential output will be the result of subtracting the negative input signal (Anti-Stokes band) to the positive input signal (Stokes band).

For a given position $z$ along the fiber, the signal change recorded in the Stokes band will be proportional to $+g_{B} P_{P}(z) P_{S}(z) \Delta z$, where $g_{B}$ is the Brillouin gain coefficient, $P_{P}$ is the pump pulse power, $P_{S}$ is the Stokes wave power and $\Delta z$ is the pulse length. Conversely, in the anti-Stokes band, the signal change will be proportional to $-g_{B} P_{P}(z) P_{A S}(z) \Delta z$ where $P_{A S}$ is the anti-Stokes power. After the balanced detection scheme, the overall signal change will be proportional to $+g_{B} P_{P}(z)\left(P_{S}(z)+P_{A S}(z)\right) \Delta z$. In typical conditions (when an external Mach-Zehnder electro-optic modulator is employed), $P_{S}=P_{A S}$, and the detected signal will be naturally doubled 


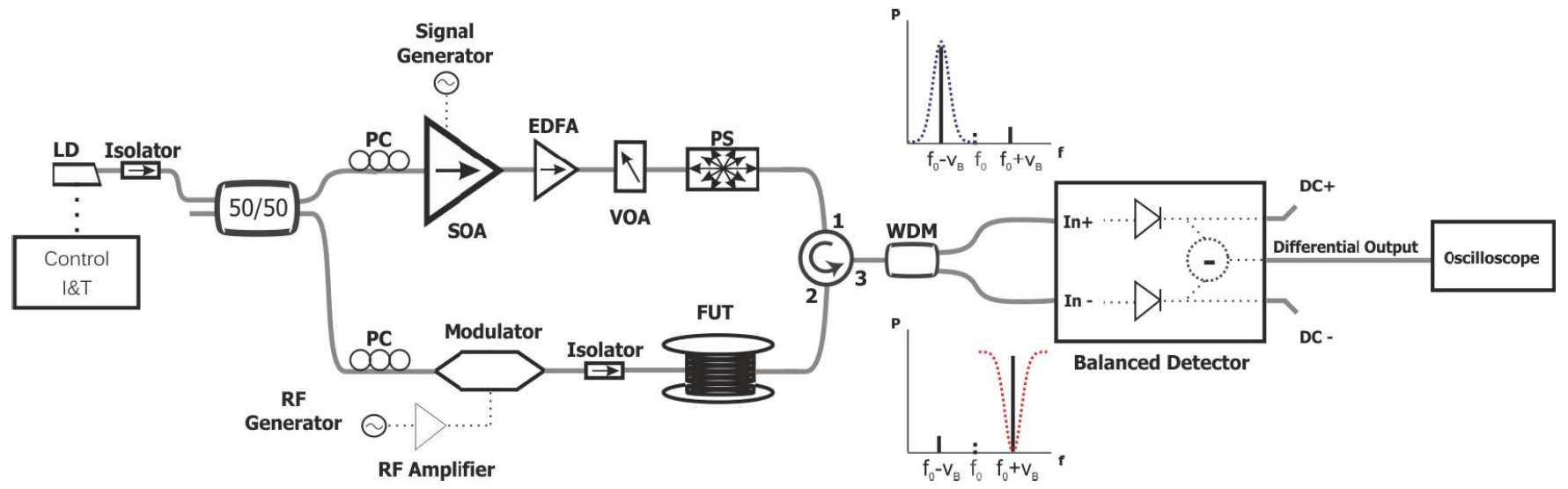

Fig. 1. Experimental setup of the BOTDA with Balanced Detection. LD: Laser Diode; PC: Polarization controller; EDFA: Erbium Doped Fiber Amplifier; RF: Radio-frequency generator; VOA: Variable Optical Attenuator; PS: Polarization Scrambler; WDM: Wavelength Division Multiplexer.

in comparison with a single-detector scheme of equal responsivity.

It is interesting now to think what happens in terms of the noise. Considering equal noise characteristics for the two input photodiodes, the use of balanced detection will lead to an increase of the noise of only $\sqrt{ } 2$ (sum of two independent equally-distributed random variables). Thus, an overall improvement of $\sqrt{ } 2$ in SNR should be expected in this configuration over a conventional single-detector option.

In addition to the previous discussion, this detection technique has an additional number of advantages: in the first place, it makes the system strongly robust to common-mode noise sources (noise sources affecting equally both bands). Common noise sources may include: master laser intensity noise, modulator drifts, varying coupling losses in the fiber, etc. However, effective common-mode intensity noise cancellation will occur solely when optical path lengths for both sidebands are equal before reaching the detector. In addition, the use of DC-coupled balanced detection allows for the amplification of the signal to higher values compared to DC-coupled single sideband detection, due to the fact that the DC levels of both independent signals are subtracted previous to the amplifying stage at the receiver, which means that the receiver clamps at a higher value of signal amplitude.

\section{EXPERIMENTAL SETUP}

To prove the above advantages in a conventional BOTDA, we developed the BOTDA scheme represented in Fig. 1. It is a variation of the classical BOTDA system [13] but incorporating balanced detection.

As in most BOTDA setups, pump and probe waves are developed from a single distributed feed-back (DFB) laser diode. A Mach-Zehnder Electro-Optic Modulator (EOM) is used to make a dual sideband with suppressed carrier (DSBSC) modulation. The extinction ratio of the EOM is $>40 \mathrm{~dB}$, thus it is possible to nearly eliminate the carrier signal. The probe power fed into the fiber is $\sim 330 \mu \mathrm{W}$ on each sideband. The modulating frequency of the EOM is controlled through an RF Generator. The RF frequency is chosen to sweep around the Brillouin Frequency Shift of the fiber under test.
At the pump side, the signal is pulsed using a Semiconductor Optical Amplifier (SOA). The SOA allows to shape high extinction ratio $(>30 \mathrm{~dB})$ optical pulses. The pulse widths used in the experiment are $40 \mathrm{~ns}$ (which implies that the sensor has 4 meter resolution), and the pulse peak power provided is $\sim 250 \mathrm{~mW}$. After pulsing, the optical signal will be again amplified through an Erbium Doped Fiber Amplifier (EDFA). The power of the pulses is controlled through a Variable Optical Attenuator (VOA) and their polarization is scrambled by a fast polarization scrambler. Although this scrambler is very fast (5 MHz of scrambling rate), the scrambling of the polarization state was imperfect, leading to some polarization noise in the trace as we will see in the results section.

After going through the fiber and experiencing Brillouin Scattering, the signal is split using a DWDM filter, which will separate Brillouin Gain band (Stokes) and Brillouin Loss band (Anti-Stokes). The filter used is sharp enough to separate both sidebands correctly, attenuating the rejected band in $>13 \mathrm{~dB}$. These two different bands are then fed to the positive and negative ports of the balanced detection system. Ultimately, the Balanced Detection System will provide three different output signals: the Differential Output (with the signal of interest), and two monitoring outputs, where we will read the DC levels of each of the input signals.

\section{Normalization Process}

In general, for BOTDA systems the acquired signal is a slight amplitude variation upon a significant background DC level. To obtain meaningful gain values, it is necessary to normalize the obtained signal values by the background DC level of the signal, which is a measure of the background Stokes $\left(P_{S}\right)$ or anti-Stokes power $\left(P_{A S}\right)$.

In balanced detection, the obtained signal has usually a small (close to zero) DC level, as the DC levels of the two photocurrents also get subtracted in the process. Thus, the DC level of the Differential Output cannot be used for the normalization. To obtain a correct normalization, it is necessary to monitor the DC levels of the two input signals separately and sum them. This is a measure of $P_{S}+P_{A S}$. With this value, we can normalize the levels of the Differential Output 

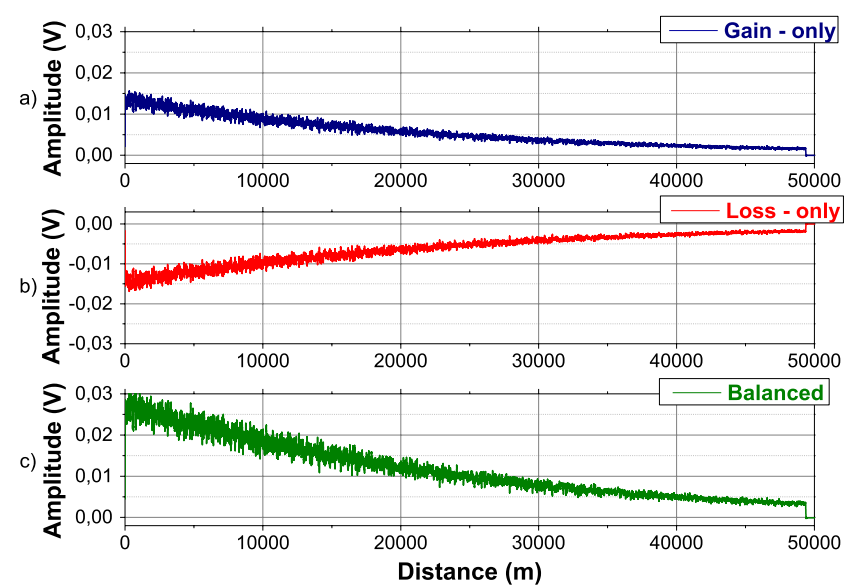

Fig. 2. Amplitude difference from the single sideband detection cases (a), (b) and balanced detection (c) for a pump-probe frequency shift of $10.883 \mathrm{GHz}$.

signal. Conventional balanced detectors typically provide these outputs, so this process is rather straightforward to do with current off-the-shelf instrumentation.

\section{RESULTS}

In this section we illustrate the results obtained with the BOTDA using the Balanced Detection configuration described previously. To obtain meaningful comparisons, the measurements have always been carried out with the same scheme, first using the gain band only (Gain-only measurements), then the loss band only (Loss-only measurements), and finally the balanced detection with both gain and loss bands (Balanced measurements). The same amount of averages is used in all cases (1024).

The measurements are performed over $\sim 50 \mathrm{~km}$ of single mode fiber (SMF) with an essentially homogeneous Brillouin frequency shift located at $10.883 \mathrm{GHz}$ at the pump wavelength $(\sim 1550 \mathrm{~nm})$.

The first remarkable result is that when acquiring in balanced mode, the trace amplitude of the Differential Output is twice the amplitude of any of the single sidebands, as illustrated in Fig. 2. This is an expected result considering the reasoning given in section II. Doubling the trace amplitude increases the signal contrast by $3 \mathrm{~dB}$, which is potentially equivalent to $15 \mathrm{~km}$ of fiber. It also leads to a theoretical $\sqrt{ } 2$ SNR increase, as shown before. In addition, balanced detection increases the robustness of the system to commonmode noises, which may affect both sidebands.

Fig. 3 shows three gain profiles at a distance of $46.818 \mathrm{~km}$, with a frequency sweep done from $10.82 \mathrm{GHz}$ to $10.95 \mathrm{GHz}$. As it can be inferred, the noise of the differential output gain profile (Fig. 3(c)) is visibly reduced compared to the gain profiles of the single Brillouin Gain (Fig. 3(a)) and Brillouin Loss (Fig. 3(b)) sidebands.

Once the frequency sweep is finished, the Brillouin Frequency Shift (BFS) is obtained by fitting a 2nd order polynomial curve to the raw gain profile, and then finding the position of the maximum of the curve. As shown in
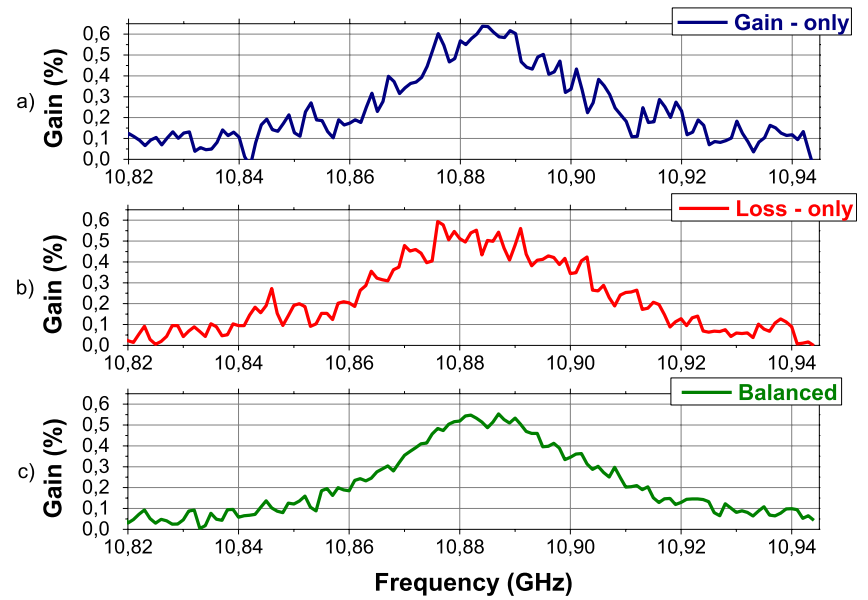

Fig. 3. Gain profiles at $46.818 \mathrm{~km}$ for a frequency sweep between $10.82 \mathrm{GHz}$ and $10.95 \mathrm{GHz}$. (a) Brillouin Gain sideband (Stokes band) acquired gain profile. (b) Brillouin Loss sideband (Anti-Stokes band) acquired gain profile. (c) Differential output acquired gain profile.

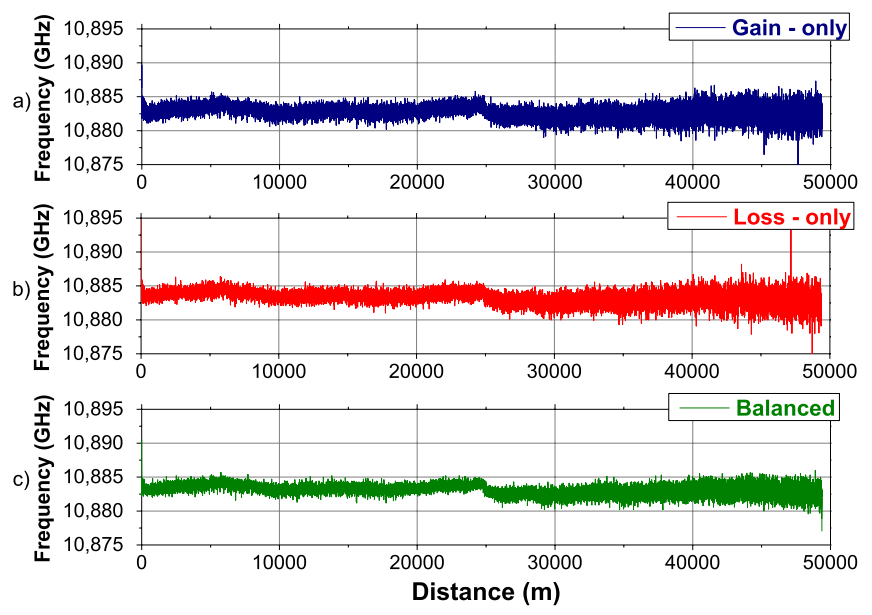

Fig. 4. Brillouin frequency shift for the single sideband acquisition cases (a), (b) and the balanced detection acquisition (c) for $50 \mathrm{~km}$ of SMF.

Fig. 4, finding the BFS at any point of the fiber is more accurate when balanced detection is employed. The standard deviation of the obtained BFS between five consecutive traces at the very beginning of the fiber (first 5000 points window) is $\sim 0.618 \mathrm{MHz}$ for both single sidebands acquisitions and $\sim 0.432 \mathrm{MHz}$ for the balanced case. The standard deviation is improved by a factor of $\sim \sqrt{ } 2$, in consistency with the SNR improvement expected in the balanced case (see [14] for details on the relationship between SNR and frequency estimation error). At the end of the fiber under test (last 5000 points window), the standard deviation for the single sideband cases is $\sim 1.232 \mathrm{MHz}$ and, as expected, for the balanced detection case it is $\sim 0.884 \mathrm{MHz}$, also resulting in an improvement ratio of $\sim \sqrt{ } 2$. In terms of the figure-of-merit discussed by [14], the FOM calculated at the end of the fiber is 25.28 for the single sideband detection cases, and raises up to 35.24 for the balanced detection case, confirming the $\sim \sqrt{ } 2$ improvement factor.

A final interesting feature of the balanced detection system is that the overall response of the system turns out to be more 


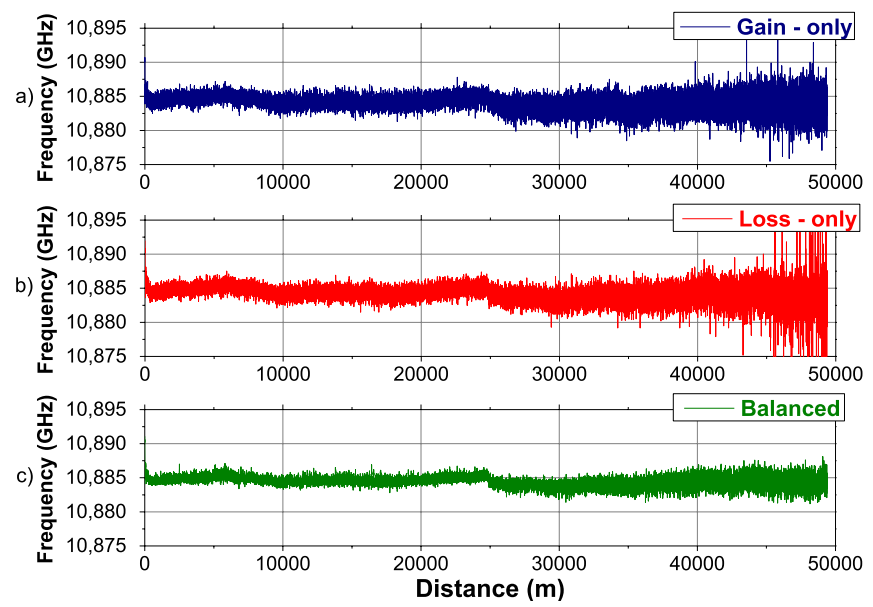

Fig. 5. Brillouin frequency shift for the single sideband acquisition cases (a), (b) and the balanced detection acquisition (c) for $\sim 50 \mathrm{~km}$ of SMF and deteriorating the Extinction Ratio of the SOA (27 dB ER).

robust to some of the variables of the experiment. One of these important variables to control is the pulse extinction ratio (ER). As stated previously, the SOA employed is electrically controlled by a signal generator. In normal conditions, it is intended to work at the highest ER that the SOA could provide. Having a high extinction ratio pulse is crucial when aiming for a good SNR. However, employing an SOA is an advanced solution and it is not always available. In exchange we can easily find conventional EOMs pulsing the pump beam, which normally have lower extinction ratio. In our case, the extinction ratio is deteriorated on purpose by varying the logical high and low voltage levels of the electrical pulse controlling the SOA.

BOTDA using balanced detection becomes more robust to the deterioration of the pulse extinction ratio. Fig. 5 shows the BFS profiles obtained for an ER of the SOA of $27 \mathrm{~dB}$ in comparison to the $34 \mathrm{~dB}$ ER case of Fig. 4.

The standard deviation of the obtained BFS between five consecutive traces calculated for the worst ER scenario achieved (27 $\mathrm{dB}$ ER) remains roughly the same at the beginning of the fiber. For the single sideband cases it is $\sim 0.625 \mathrm{MHz}$ and once again, for the balanced acquisition it is $\sim 0.438 \mathrm{MHz}$, resulting in an improvement factor of $\sim \sqrt{ } 2$. However, the standard deviation at the end of the fiber is $\sim 1.759 \mathrm{MHz}$ for the single sideband acquisitions and $\sim 0.891 \mathrm{MHz}$ for the balanced detection case, which means, the standard deviation of the obtained BFS for the single sideband cases is more than double the uncertainty of the balanced case.

For any of the single sideband acquisitions, the fitting and finding the maximum processes might lead to points with extreme error, which are often observed at the end of the fiber. This situation is caused by the appearance of extreme peaks in the gain profile at that position, which perturb the fitting process. As it can be seen, the misleading points in the fitting and finding the maximum procedure are substantially avoided in the balanced case, even using raw gain profiles.

The remarkable feature of employing balanced detection is that the standard deviation of the obtained BFS at the end of the fiber remains stable $(\sim 0.89 \mathrm{MHz})$ as the ER is worsened, showing an improved robustness to the deterioration of this experimental parameter.

\section{CONCLUSION}

In conclusion, we have presented the application of a wellknown detection technique (balanced detection) to BOTDA systems. We have shown, both theoretically and experimentally, that remarkable improvements in terms of SNR can be obtained. In typical conditions, the trace amplitude is doubled and the SNR is improved by a factor of $\sqrt{ } 2$. The system also features a strong robustness to common mode noise in both sidebands (including master laser noise, modulator drifts, etc.). Furthermore, we have proven a strong robustness to the deterioration of the pump pulse extinction ratio, which might reduce the technical requirements of future experiments.

\section{REFERENCES}

[1] X. Angulo-Vinuesa, et al., "Raman-assisted Brillouin distributed temperature sensor over $100 \mathrm{~km}$ featuring $2 \mathrm{~m}$ resolution and $1.2^{\circ} \mathrm{C}$ uncertainty," J. Lightw. Technol., vol. 30, no. 8, pp. 1060-1065, Apr. 15, 2012.

[2] M. A. Soto, P. K. Sahu, G. Bolognini, and F. Di Pasquale, "Brillouinbased distributed temperature sensor employing pulse coding," IEEE Sensors J., vol. 8, no. 3, pp. 225-226, Mar. 2008.

[3] M. A. Soto, M. Taki, G. Bolognini, and F. Di Pasquale, "Simplex-coded BOTDA sensor over 120-km SMF with 1-m spatial resolution assisted by optimized bidirectional Raman amplification," IEEE Photon. Technol. Lett., vol. 24, no. 20, pp. 1823-1826, Oct. 15, 2012.

[4] C. Jin, W. Zou, and J. Chen, "Signal-to-noise ratio improvement in Brillouin optical correlation domain analysis combining Brillouin gain and loss effects," Proc. SPIE, vol. 8421, p. 842114, Oct. 2012.

[5] P. C. D. Hobbs, "Ultrasensitive laser measurements without tears," Appl. Opt., vol. 36, no. 4, pp. 903-920, Feb. 1997.

[6] H. R. Carleton and W. T. Maloney, "A balanced optical heterodyne detector," Appl. Opt., vol. 7, no. 6, pp. 1241-1243, Jun. 1968.

[7] I. Andonovic, L. Tancevski, M. Shabeer, and L. Bazgaloski, "Incoherent all-optical code recognition with balanced detection," J. Lightw. Technol., vol. 12, no. 6, pp. 1073-1080, Jun. 1994.

[8] X. Wang, N. Wada, T. Miyazaki, and K. Kitayama, "Coherent OCDMA system using DPSK data format with balanced detection," IEEE Photon. Technol. Lett., vol. 18, no. 7, pp. 826-828, Apr. 1, 2006.

[9] T. Dennis, S. D. Dyer, and A. Dienstfrey, "Phase-dispersion light scattering for quantitative size-imaging of spherical scatterers," Proc. SPIE, vol. 6446, p. 644609, Feb. 2007.

[10] G. P. Agrawal, Nonlinear Fiber Optics, 3rd ed. San Diego, CA, USA: Academic, 2001, ch. 9.

[11] M. Niklés, L. Thévenaz, and P. A. Robert, "Simple distributed fiber sensor based on Brillouin gain spectrum," Opt. Lett., vol. 21, no. 10, pp. 758-760, May 1996.

[12] A. Minardo, R. Bernini, and L. Zeni, "A simple technique for reducing pump depletion in long-range distributed Brillouin fiber sensors," IEEE Sensors J., vol. 9, no. 6, pp. 633-634, Jun. 2009.

[13] X. Angulo-Vinuesa, S. Martín-López, P. Corredera, and M. Gonzalez-Herraez, "Raman-assisted Brillouin optical time-domain analysis with sub-meter resolution over $100 \mathrm{~km}$," Opt. Express, vol. 20, no. 11, pp. 12147-12154, May 2012.

[14] L. Thévenaz and M. A. Soto, "Rating the performance of a Brillouin distributed fiber sensor," Proc. SPIE, vol. 8421, p. 8421A7, Oct. 2012. 